\title{
Energy-Efficient Resource Allocation in SWIPT Enabled NOMA Systems
}

\author{
Jie Tang*, Jingci Luo*, Daniel So ${ }^{\dagger}$, Emad Alsusa ${ }^{\dagger}$, Kai-Kit Wong ${ }^{\ddagger}$, Nan Zhao $^{\S}$ \\ ${ }^{*}$ School of Electronic and Information Engineering, South China University of Technology, China \\ ${ }^{\dagger}$ School of Electrical and Electronic Engineering, University of Manchester, UK \\ ${ }_{\ddagger}^{\ddagger}$ Department of Electronic and Electrical Engineering, University College London, UK \\ ${ }^{\S}$ School of Information and Communication Engineering, Dalian University of Technology, China
}

\begin{abstract}
In this paper, we investigate joint power allocation and time switching (TS) control for energy efficiency (EE) optimization in a TS-based simultaneous wireless information and power transfer (SWIPT) non-orthogonal multiple access (NOMA) system. Our aim is to optimize the EE of the system whilst satisfying the constraints on maximum transmit power, minimum data rate and minimum harvested energy per-terminal. The considered EE optimization problem is formulated and then transformed according to the duality of broadcast channels (BC) and multiple access channels (MAC). The corresponding non-linear and non-convex optimization problem, involving joint optimization of power allocation and time switching factor, is difficult to solve directly. In order to tackle this problem, we develop a dual-layer algorithm where a convex programmingbased Dinkelbach's method is proposed to optimize the power allocation in the inner-layer and an efficient search method is then applied to optimize the TS factor in the outer-layer. Numerical results validate the theoretical findings and demonstrate that significant performance gain over orthogonal multiple access (OMA) scheme in terms of EE can be achieved by the proposed algorithm in a SWIPT-enabled NOMA system.
\end{abstract}

\section{INTRODUCTION}

The exponential growth of wireless data services driven by mobile Internet and connected devices has triggered the investigation of fifth generation $(5 \mathrm{G})$ cellular networks. However, the available spectrum resources are far from enough to support the communication systems with the increasing demand for high data rate. This trend makes spectral efficiency (SE) to be one of the main performance indicator for the design and optimization of wireless systems. A prominent strategy for improving the SE is non-orthogonal multiple access (NOMA), where each subchannel is allowed to serve multiple terminals at the same time [1], and hence it has received considerable attention as a promising candidate for $5 \mathrm{G}$ [2].

On the other hand, the pursuit of ever higher data rate constitutes to ever-rising network power consumption which has severe implications in terms of both economic and ecological costs, and thus energy saving has been recognized as an urgent issue worldwide. In order to meet these requirements, the evolving $5 \mathrm{G}$ cellular wireless networks are envisioned to provide higher efficiency of resource utilization, including both SE and energy efficiency (EE) [3]. The recent progress in the research on wireless power transfer (WPT) provides possibility of improving EE [4]. Furthermore, it is known that the radio frequency (RF) signals are the carriers of both information and energy, which makes it possible to combine WPT and wireless information transmission (WIT) in wireless communication systems. Motivated by this, an advanced technology named simultaneous wireless information and power transfer (SWIPT), has emerged recently in [5], aiming to save energy as well as prolong the battery-life of devices by achieving the parallel transmission of information and energy. As a result, SWIPT is regarded as a potential energy-efficient solution for the forthcoming $5 \mathrm{G}$, which has attracted extensive attention in both academia and industry.

With the extensive fundamental studies on both SWIPT and NOMA, the combination of these two techniques has aroused great interest recently and it was verified that combining SWIPT with NOMA could bring plenty of advantages, i.e., user fairness [6], data rate [7], secrecy rate [8], etc. However, its impact on the EE performance has not been investigated, which is still an open question. Unlike the rate or secrecy rate optimization problem in existing literatures which may not be energy-efficient, the EE optimization problem requires the balancing of the achievable rate and the overall power consumption. Motivated by this, we consider joint power allocation and time switching (TS) control for QoS-constrained EE optimization in the downlink of SWIPT-enabled NOMA systems, where maximum transmit power constraint, minimum rate constraints and minimum harvested energy constraints are taken into consideration.

\section{System Model AND Problem Formulation}

In this section, the SWIPT-enabled NOMA system model with TS-based receivers is presented firstly. Then we formulate the EE optimization problem mathematically.

\section{A. System Model}

The considered SWIPT-enabled NOMA system with TSbased terminals includes one base station (BS) and $K$ different terminals; all are equipped with single antenna. The total transmit power of the $\mathrm{BS}$ is limited to $P_{\max }$. We assume the power allocated to the $k$ th terminal in NOMA system is $P_{k}$. Furthermore, every terminal consists of an information decoding (ID) circuit and an energy harvesting (EH) rectification circuit. TS scheme is adopted to achieve ID and EH 
in two orthogonal time slots. Let $\alpha$ denotes the portion of transmission time allocated to the ID time slot, and thus, $1-\alpha$ corresponds to that for the EH time slot.

Since all the terminals are sharing the same bandwidth $(B)$, they will interfere with each other when decoding information. Here successive interference cancellation (SIC) is applied to reduce mutual interference and improve the performance of the system. It has been shown in [9] that the capacity region of broadcast channels can be achieved when successive decoding in the order of increasing channel gains is applied. Without loss of generality, we assume that the channel gains satisfy the following condition: $h_{1} \geq h_{2} \geq \ldots \geq h_{K}$. In order to achieve the capacity boundary, the decoding order is set to $(K, K-1, \cdots, 1)$ in which the $K^{t h}$ terminal is decoded first. Channel state information (CSI) is assumed to be perfectly known at the BS and all the terminals. Note that the CSI at the receivers can be obtained from the channel estimation of the downlink pilots, while CSI at the transmitter can be acquired via uplink channel estimation in time division duplex (TDD) mode.

Based on the system model presented above, the achievable data rate of the $k^{\text {th }}$ terminal can be denoted as

$$
R_{k}=\alpha B \log _{2}\left(1+\frac{P_{k} h_{k}}{\sigma^{2}+h_{k} \sum_{i=1}^{k-1} P_{i}}\right),
$$

where $\sigma^{2}$ denotes the power of the additive Gaussian white noise (AWGN).

On the other hand, the power harvested by the $k^{t h}$ terminal can be expressed as

$$
E_{k}=(1-\alpha) \eta h_{k} \sum_{k=1}^{K} P_{k}
$$

where $\eta$ is the power conversion efficiency of the EH receivers.

The total data rate and harvested power of the system can thus be expressed as

$$
\begin{aligned}
& R_{\text {total }}=\sum_{k=1}^{K} R_{k} \\
& E_{\text {total }}=\sum_{k=1}^{K} E_{k} .
\end{aligned}
$$

Generally, the power consumption of the conventional wireless communication system is defined as the sum of the total power transmitted by the transmitters and the power consumed by the system hardware to complete the communication process, i.e.,

$$
P_{\text {totall }}=\zeta \sum_{k=1}^{K} P_{k}+P_{C},
$$

where $\zeta$ accounts for the reciprocal of the power amplifier drain efficiency, $P_{C}$ denotes the power consumed by the system hardware (circuit power).

Different from the conventional wireless communication system, the power consumption in a SWIPT system can be compensated by the transferred energy, i.e., harvested power at all the receivers. As a result, similar to the approach in [10], the total system power consumption is given by

$$
P_{\text {total }}=\zeta \sum_{k=1}^{K} P_{k}+P_{C}-E_{\text {total }} .
$$

\section{B. Problem Formulation}

According to [11], the EE of the system can be defined as the ratio of the total achievable rate and the total power consumption of the communication system. Therefore, the EE of the considered SWIPT-enabled NOMA system with TSbased receiver can be formulated as follow

$$
\begin{aligned}
\eta_{E E} & =\frac{R_{\text {total }}}{P_{\text {total }}} \\
& =\frac{\alpha B \sum_{k=1}^{K} \log _{2}\left(1+\frac{P_{k} h_{k}}{\sigma^{2}+h_{k} \sum_{i=1}^{k-1} P_{i}}\right)}{\left(\zeta-(1-\alpha) \eta \sum_{k=1}^{K} h_{k}\right) \sum_{k=1}^{K} P_{k}+P_{C}} .
\end{aligned}
$$

The objective of this work is to maximize the EE of the TS-based SWIPT-enabled NOMA system with the constraints of total transmitted power obtained from BS as well as the quality of service (QoS) demanded by the terminals, including the minimum data rate constraints and the minimum harvested energy per terminal. Hence, the EE optimization problem can be mathematically formulated as follows

$$
\begin{array}{ll}
\max _{\alpha, P_{k}} & \frac{\alpha B \sum_{k=1}^{K} \log _{2}\left(1+\frac{P_{k} h_{k}}{\sigma^{2}+h_{k} \sum_{i=1}^{k-1} P_{i}}\right)}{\left(\zeta-(1-\alpha) \eta \sum_{k=1}^{K} h_{k}\right) \sum_{k=1}^{K} P_{k}+P_{C}} \\
\text { s.t. } \quad & \alpha B \log _{2}\left(1+\frac{P_{k} h_{k}}{\sigma^{2}+h_{k} \sum_{i=1}^{k-1} P_{i}}\right) \geq R_{\text {min }}, \\
& \forall k \in \mathcal{K}, \\
& (1-\alpha) \eta h_{k} \sum_{k=1}^{K} P_{k} \geq E_{\min }, \forall k \in \mathcal{K}, \\
& \sum_{k=1}^{K} P_{k} \leq P_{\max }, \\
& 0 \leq \alpha \leq 1 .
\end{array}
$$

where $\mathcal{K}=\{1,2, \ldots, K\}$ denotes the set of all terminals. Equation (9) corresponds to the minimum rate constraint for each ID receiver, and (10) corresponds to the minimum harvested power constraints for each $\mathrm{EH}$ receiver. $R_{\min }$ and $E_{\text {min }}$ denote the minimum rate target and the minimum harvested power requirement respectively. In (11), the total power budget is set to $P_{\max }$, while constraint (12) limits the range of the time switching factor to $[0,1]$.

We can observe that the objective function (8) is a rather complicated fraction, and thus it is a non-linear optimization problem. Besides, the problem involves two sets of variables, i.e., the time switching factor $\alpha$ and transmitted power allocation $P_{k}$. Jointly optimizing the two variables makes the optimization (8)-(12) non-convex and therefore difficult to obtain the optimal solution directly. In the following sections, we will develop resource allocation schemes to jointly optimize $\alpha$ and $P_{k}$ in order to obtain the maximum EE. To overcome the difficulty, as stated in [12], for any optimization problems 
with multiple variables, we can analyze and solve the problem over some variables, regarding the rest as constants; and then solve the problem over the remaining variables. Therefore, we will separate the optimization procedure of $P_{k}$ and $\alpha$. In other word, we firstly optimize $P_{k}$ with a fixed $\alpha$, and then turn to deal with $\alpha$ with the updated power allocation.

\section{Joint Power Allocation And Time Switching CONTROL AlgORITHM}

In this section, we consider the joint power allocation and time switching control for the SWIPT-enabled NOMA system with multiple TS-based receivers.

\section{A. Transformation of the Optimization Problem}

The objective function given in (8) is complicated due to its unwieldy summation form of numerator. Hence, we develop a transformation method to simplify the optimization problem, according to the duality of BC and MAC. Specifically, for the considered downlink SWIPT-enabled NOMA system (BC), the expression of achievable rate and harvested power given in (1) and (2), as well as the optimization problem given in (8)-(12), can be rewritten by replacing $P_{k}, R_{k}, E_{k}$ using $P_{k}^{B}, R_{k}^{B}$ and $E_{k}^{B}$ respectively.

According to [9], the duality of $\mathrm{BC}$ and $\mathrm{MAC}$ is expressed as follow: i) The channel bandwidth of $\mathrm{BC}$ is equivalent to the one of MAC. ii) The channel gain from BS to the $k^{t h}$ terminal in $\mathrm{BC}$ is equivalent to the one from the $k^{t h}$ terminal to $\mathrm{BS}$ in MAC, both denoted as $h_{k}$. iii) The total power allocated to all terminals by $\mathrm{BS}$ in $\mathrm{BC}$ is equivalent to the total power transmitted to $\mathrm{BS}$ by all terminals in MAC, i.e,

$$
\sum_{k=1}^{K} P_{k}^{B}=\sum_{k=1}^{K} P_{k}^{M},
$$

where $P_{k}^{M}$ denotes the power transmitted by the $k^{t h}$ terminal in MAC. iv) When SIC technique is adopted, the ID order in $\mathrm{BC}$ is opposite to the one in MAC. v) The power allocated to the $k^{t h}$ terminal $P_{k}^{B}$ in $\mathrm{BC}$ and the power transmitted by the $k^{t h}$ terminal $P_{k}^{M}$ are related to each other as follows

$$
P_{k}^{B}=P_{k}^{M} \cdot \frac{\sigma^{2}+h_{k} \sum_{i=1}^{k-1} P_{i}^{B}}{\sigma^{2}+\sum_{i=k+1}^{K} h_{i} P_{i}^{M}} .
$$

Based on the duality described above, the achievable sum rate in the $\mathrm{BC}$ is equivalent to that of $\mathrm{MAC}$, which is expressed as follows

$$
\begin{aligned}
R_{k}^{B}=R_{k}^{M} & =\alpha B \log _{2}\left(1+\frac{h_{k} P_{k}^{M}}{\sigma^{2}+\sum_{i=k+1}^{K} h_{i} P_{i}^{M}}\right), \\
\sum_{k=1}^{K} R_{k}^{B} & =\sum_{k=1}^{K} R_{k}^{M} \\
& =\sum_{k=1}^{K} \alpha B \log _{2}\left(1+\frac{h_{k} P_{k}^{M}}{\sigma^{2}+\sum_{i=k+1}^{K} h_{i} P_{i}^{M}}\right) \\
& =\alpha B \log _{2}\left(1+\frac{\sum_{k=1}^{K} h_{k} P_{k}^{M}}{\sigma^{2}}\right) .
\end{aligned}
$$


1) Initialize $q=0$, and $\Delta$ as the stopping criterion;

2) REPEAT

3) For a given $q$, solve (26)-(29) to obtain the power allocation $\boldsymbol{P}^{M}$;

4) $\quad$ IF $U_{R}\left(\boldsymbol{P}^{\boldsymbol{M}}\right)-q U_{T}\left(\boldsymbol{P}^{\boldsymbol{M}}\right) \leq \Delta$

5) $\quad$ Convergence $=$ TRUE;

6) RETURN $P^{M^{*}}=P^{M}$;

$$
q^{*}=\frac{U_{R}\left(\boldsymbol{P}^{M}\right)}{U_{T}\left(\boldsymbol{P}^{M}\right)} ;
$$

7) ELSE

8) Set $q=\frac{U_{R}\left(\boldsymbol{P}^{M}\right)}{U_{T}\left(\boldsymbol{P}^{M}\right)}$ and $n=n+1$,

9) END IF

Convergence $=$ FALSE;

10) UNTIL Convergence $=$ TRUE.

TABLE I: Proposed iterative power allocation algorithm based on Dinkelbach's method

function into a subtractive form of numerator and denominator, and the iterative algorithm based on Dinkelbach's method is concluded in Table I, where we let

$$
\begin{aligned}
& U_{R}\left(\boldsymbol{P}^{\boldsymbol{M}}\right)=\alpha_{0} B \log _{2}\left(1+\frac{\sum_{k=1}^{K} h_{k} P_{k}^{M}}{\sigma^{2}}\right), \\
& U_{T}\left(\boldsymbol{P}^{\boldsymbol{M}}\right)=\left(\zeta-\left(1-\alpha_{0}\right) \eta \sum_{k=1}^{K} h_{k}\right) \sum_{k=1}^{K} P_{k}^{M}+P_{C} .
\end{aligned}
$$

As it can be seen from Table I, the crucial step for the developed Dinkelbach's method-based algorithm is to deal with the following equivalent optimization problem with a given $q$ (step 3 in Table I)

$$
\begin{aligned}
\max _{P_{k}^{M}} & \alpha_{0} B \log _{2}\left(1+\frac{\sum_{k=1}^{K} h_{k} P_{k}^{M}}{\sigma^{2}}\right) \\
& -q\left(\left(\zeta-\left(1-\alpha_{0}\right) \eta \sum_{k=1}^{K} h_{k}\right) \sum_{k=1}^{K} P_{k}^{M}+P_{C}\right) \\
\text { s.t. } & \alpha_{0} B \log _{2}\left(1+\frac{h_{k} P_{k}^{M}}{\sigma^{2}+\sum_{i=k+1}^{K} h_{i} P_{i}^{M}}\right) \geq R_{\text {min }} \\
& \forall k \in \mathcal{K}, \\
& \left(1-\alpha_{0}\right) \eta h_{k} \sum_{k=1}^{K} P_{k}^{M} \geq E_{\min }, \forall k \in \mathcal{K} \\
& \sum_{k=1}^{K} P_{k}^{M} \leq P_{\max } .
\end{aligned}
$$

Proposition 1: For a fixed parameter $q$, the objective function (26) is strictly concave in $P_{k}^{M}(\forall k \in \mathcal{K})$.

Since the objective function is a concave function and the constraints form a convex set, the equivalent optimization problem in (26)-(29) is a standard form of convex optimization problem, which can be solved by standard numerical methods such as the interior-point method [14]. Therefore, the transformed optimization problem (19)-(23) (given $\alpha$ ) can be solved by the proposed iterative power allocation algorithm based on the Dinkelbach's method in Table I.
C. Time Switching Factor Assignment under Fixed Power Allocation

Similar to the works in OMA systems, e.g. MIMO systems [11] and OFDMA systems [15], an exhaustive search method can be applied here to find the optimal time switching factor $\alpha$. However, the exhaustive search method is computationally complicated, which depends on the iteration step of $\alpha$. It is true that the complexity decreases with increasing iteration step, however, it will bring a negative impact on the accuracy of the result. Motivated by this, we develop an efficient searching algorithm for obtaining the optimal time switching factor according to the relationship between $\mathrm{EE}$ and the time switching factor $\alpha$.

Assuming that we have obtained an optimal power allocation $\boldsymbol{P}^{M}$ from the last iteration, we attempt to obtain the optimal time switching factor $\alpha$ based on a fixed $\boldsymbol{P}^{M}$, and hence the problem in (19)-(23) is accordingly converted to

$$
\begin{aligned}
\max _{\alpha} & \frac{\alpha B \log _{2}\left(1+\frac{\sum_{k=1}^{K} h_{k} P_{k}^{M}}{\sigma^{2}}\right)}{\left(\zeta-(1-\alpha) \eta \sum_{k=1}^{K} h_{k}\right) \sum_{k=1}^{K} P_{k}^{M}+P_{C}} \\
\text { s.t. } \quad & \alpha B \log _{2}\left(1+\frac{h_{k} P_{k}^{M}}{\sigma^{2}+\sum_{i=k+1}^{K} h_{i} P_{i}^{M}}\right) \geq R_{\text {min }} \\
& \forall k \in \mathcal{K}, \\
& (1-\alpha) \eta h_{k} \sum_{k=1}^{K} P_{k}^{M} \geq E_{\text {min }}, \forall k \in \mathcal{K} \\
& 0 \leq \alpha \leq 1 .
\end{aligned}
$$

To develop an efficient searching algorithm for obtaining the optimal TS factor, we investigate the relationship between $\mathrm{EE}$ and the TS factor $\alpha$, which is summarized in the following Proposition.

Proposition 2: With a fixed power allocation $\boldsymbol{P}^{M}$ that satisfies the constraints in (27)-(29), EE is monotonically increasing or decreasing with respect to the TS factor $\alpha$.

Based on this observation, we first decide the trend of EE according to the optimal power allocation $\boldsymbol{P}^{M}$ obtained in the last step. Meanwhile, it's not difficult to derive the feasible region of $\alpha$ based on the constraints (31)-(33). Therefore, for the case that $\mathrm{EE}$ is monotonically increasing in $\alpha$, we obtain the corresponding optimal time switching factor $\alpha^{\star}$ at the maximum available $\alpha$ that meets the QoS demands. On the contrary, the optimal time switching factor $\alpha^{\star}$ is set to the minimum available value for the case of decreasing trend.

\section{Joint Power Allocation and Time Switching Solution}

The joint power allocation and time switching strategy for the transformed problem (19)-(23) is written as follows

$$
\begin{aligned}
& \underbrace{\alpha[0] \rightarrow \boldsymbol{P}^{\boldsymbol{M}^{\star}}[0], \eta_{E E}^{\star}[0]}_{\text {Initialization }} \rightarrow \cdots \rightarrow \underbrace{\alpha[t] \rightarrow \boldsymbol{P}^{\boldsymbol{M}^{\star}}[t], \eta_{E E}^{\star}[t]}_{\text {Iteraetion t }} \\
& \rightarrow \cdots \rightarrow \underbrace{\alpha\left[t_{\max }\right] \rightarrow \boldsymbol{P}^{\boldsymbol{M}^{\star}}\left[t_{\max }\right], \eta_{E E}^{\star}\left[t_{\max }\right]}_{\text {Iteraetion } t_{\max }}
\end{aligned}
$$

where $t_{\max }$ denotes the maximum number of iteration. With the developed power allocation algorithm and TS control 


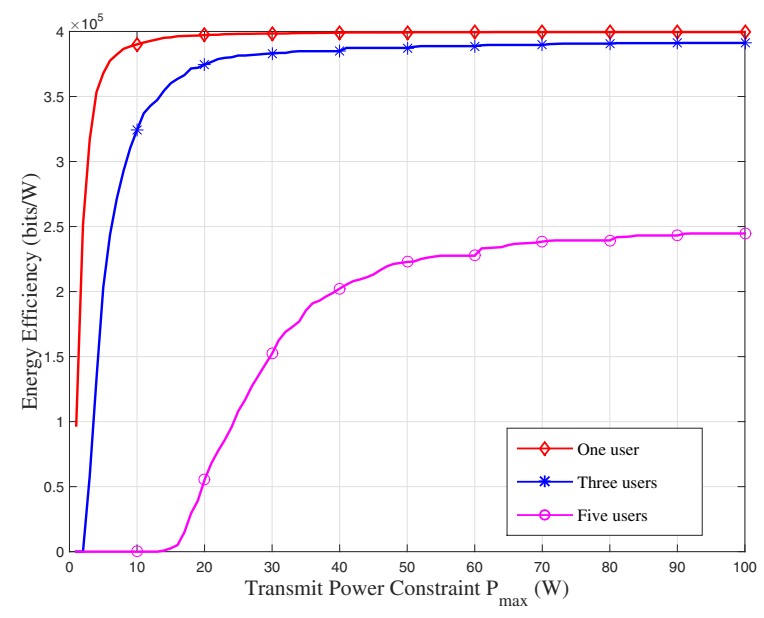

Fig. 1: The performance of the proposed algorithm with different power constraints (EE vs transmitted power constraint).

strategy, the updated process is repeated until no further improvement can be obtained.

Therefore, the algorithm about joint power allocation and time switching control for the transformed dual MAC problem (19)-(23) has been developed completely. We denote the optimal power allocation and the optimal TS factor as $\boldsymbol{P}^{M^{o p t}}$ and $\alpha^{o p t}$ respectively. In order to further obtain the optimal resource allocation scheme for the original downlink problem, including the time switching factor and the power allocation, we just need to transform $\boldsymbol{P}^{M^{o p t}}$ into $\boldsymbol{P}^{\boldsymbol{B}^{\text {opt }}}$ using (14). Besides, the optimal time switching factor remains $\alpha^{o p t}$. Therefore, the original optimization problem (8)-(12) can be successfully tackled.

\section{Simulation Results}

In this section, numerical results are presented to demonstrate the performance of the theoretical findings and the superiority of the proposed algorithms in terms of EE. The drain efficiency of the power amplifier $\zeta$ is set to $38 \%$ whereas the $\mathrm{EH}$ efficiency is taken to be $\eta=50 \%$. In addition, the bandwidth of the system is set to $B=1 M H z$. The static circuit power at the transmitter $P_{C}$ is assumed to be $10 \mathrm{~W}$.

Firstly, the EE performance of the proposed joint power allocation and time switching control algorithm with various QoS demands are evaluated. In these simulations, the circuit power is fixed to $P_{C}=10 \mathrm{~W}$ and the number of terminals is set to one, three and five for comparison. We first investigate the EE performance of the proposed solution with different power budgets. In particular, we set the total transmit power within a range of $1 \leq P_{\max } \leq 100 \mathrm{~W}$, and the minimum rate constraint and harvested energy constraint are set to $R_{\text {min }}=2 \mathrm{Mbits} / \mathrm{s}$ and $E_{\min }=0.2 \mathrm{~W}$ respectively. As shown in Fig. 1, the optimal EE achieved by the proposed scheme are monotonically non-decreasing in the total transmit power constraint. Particularly, the EE increases dramatically with a smaller power budget, and then approaches an asymptotic value where a balance among the achievable rates and the total power consumption is obtained. In other words, the additional power budget does not constitute to extra gain in EE. Besides,

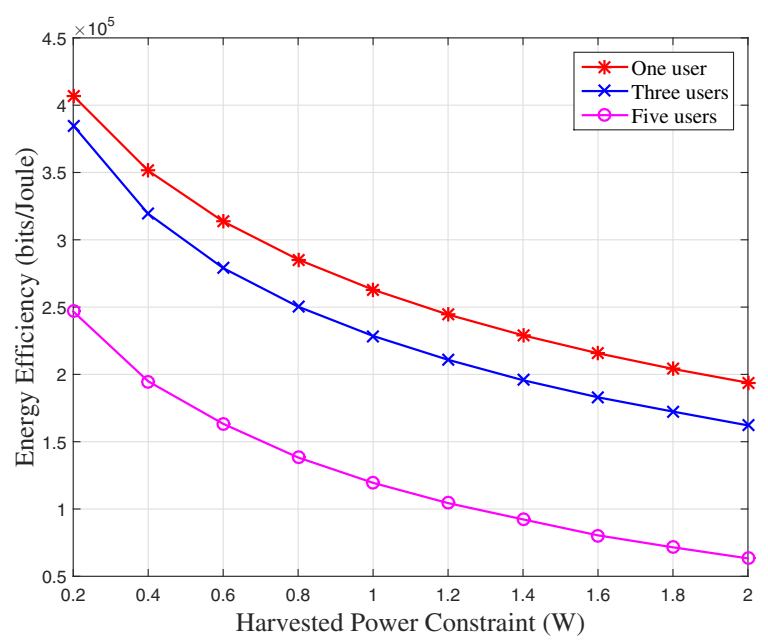

Fig. 2: The performance of the proposed algorithm with different harvested power constraints (EE vs harvested power constraint).

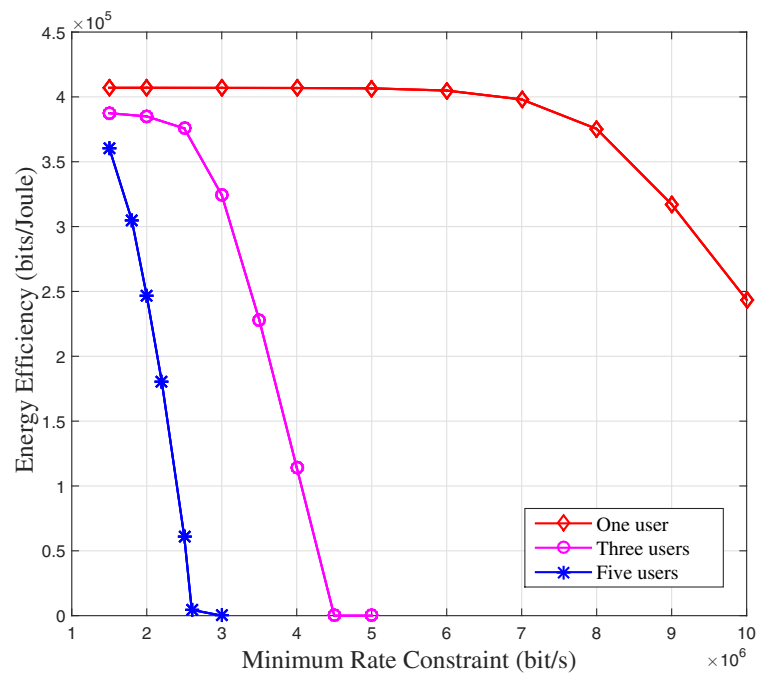

Fig. 3: The performance of the proposed algorithm with different minimum rate constraints (EE vs minimum rate constraint).

if there are more terminals in the network, the maximum power budget $P_{\max }$ to reach the maximum EE is required to be higher. These two results make sense when we expect to achieve better EE performance with a limited power.

We next show in Fig. 2 and Fig. 3 the optimal EE under different rate and harvested energy requirements. It can be seen in Fig. 2 that the optimal EE drops as the minimum demanded harvested power increases. To satisfy a higher minimum harvested energy demand, extra power is required. It has been shown in Fig. 1 that this additional power cannot provide any EE gain. More precisely, this EE loss is mainly due to the increased energy allocated to $\mathrm{EH}$, which leads to an imbalance between the numerator (transmission rate) and the denominator (power consumption) of the EE metric. A similar trend can also be seen for the case of varying the minimum rate constraint, where the optimal EE achieved by the proposed approach remains unchanged up to a certain minimum transmission rate requirement, but decreases 


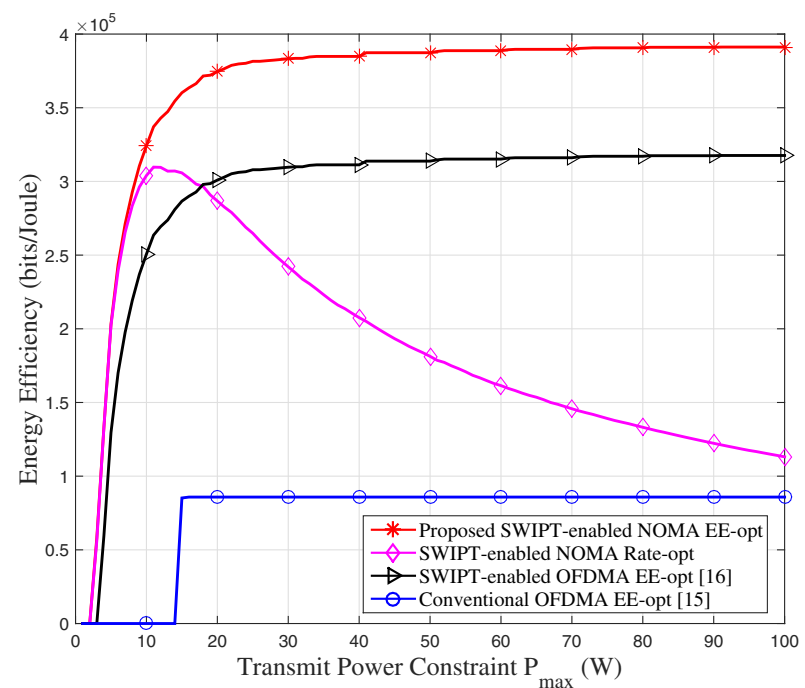

Fig. 4: Impact of the maximum transmit power on the EE performance under different resource allocation approaches.

thereafter. Additionally, we can draw a conclusion that $\mathrm{EE}$ is non-increasing with respect to the number of terminals from Fig. 1, Fig. 2 and Fig. 3. The reason for this result is that the ID receivers will interfere with each other when decoding information in NOMA system, and hence more transmit power is required to obtain the data rate per terminal.

Finally, we compare the EE performance among different approaches. The curve marked as "Proposed SWIPT-enabled NOMA EE-opt" denotes the EE performance of the proposed joint power allocation and TS control scheme for SWIPTenabled NOMA system, and the curve "SWIPT-enabled NOMA Rate-opt" represents the sum rate maximization scheme in the same system. It can be observed that with the increasing total transmit power budget $P_{\max }$, the EE achieved by "SWIPT-enabled NOMA Rate-opt" first increases and then decreases with $P_{\max }$. That is because for the rate maximization approach, the whole transmit power will be allocated for all the terminals to obtain the maximum data rate. However, when the transmit power is increased to a certain extent, the effectiveness on the improvement of data rate is not significant due to the property of log function. Therefore, the achieved EE drops after certain power level. On the other hand, since the additional power budget does not contribute to extra gain in $\mathrm{EE}$, the EE achieved by the proposed joint power allocation and time switching control algorithm is monotonically nondecreasing in the total transmit power constraint $P_{\max }$. In addition, the EE performance of OMA system is also studied. In particular, we take into account the approach that maximize the EE in SWIPT-enabled OFDMA system [16] and the approach that maximize the EE in a conventional OFDMA system [15], which are marked as "SWIPT-enabled OFDMA EE-opt" and "conventional OFDMA EE-opt" respectively. It is clear that the EE achieved by the proposed SWIPT-NOMA scheme outperforms both the SWIPT-OFDMA scheme and the conventional OFDMA-based scheme, which has demonstrated the applicability of our proposed approach and confirmed the advantages of integration SWIPT into NOMA system.

\section{CONCLUSIONS}

In this paper, we have addressed the EE optimization problem for SWIPT-enabled NOMA systems with TS-based receiver. The corresponding EE optimization problem, which involves joint optimization of the TS factor and power allocation, is non-convex and thus the solution is non-trivial and cannot be obtained directly. In order to obtain a feasible solution for this problem, we develop a dual-layer iterative resource allocation algorithm based on the principle of BC-MAC duality. In particular, a convex programming-based Dinkelbach's method has been developed to obtain the optimal power allocation for the inner-layer, whilst a novel TS control strategy has been proposed for the outer-layer. Numerical results demonstrate the EE performance of the proposed strategy of jointly optimizing the power allocation and the TS factor. More importantly, compared to the conventional OMA approach, our findings have illustrated that significant EE gain can be achieved by our proposed algorithm, and this has confirmed the advantages of integration SWIPT into NOMA systems.

\section{REFERENCES}

[1] L. Dai, B. Wang, Y. Yuan, S. Han, C. 1. I, and Z. Wang, "Non-orthogonal multiple access for 5G: solutions, challenges, opportunities, and future research trends," IEEE Commun. Mag., vol. 53, no. 9, pp. 74-81, 2015.

[2] L. Zhang, M. Xiao, G. Wu, M. Alam, Y. C. Liang, and S. Li, "A survey of advanced techniques for spectrum sharing in 5G networks," IEEE Wireless Commun., vol. 24, no. 5, pp. 44-51, Oct. 2017.

[3] J. G. Andrews, S. Buzzi, W. Choi, S. V. Hanly, A. Lozano, A. C. K. Soong, and J. C. Zhang, "What will 5G be?" IEEE Journal on Sel. Areas in Commun., vol. 32, no. 6, pp. 1065-1082, June 2014.

[4] X. Lu, P. Wang, D. Niyato, D. I. Kim, and Z. Han, "Wireless networks with RF energy harvesting: A contemporary survey," IEEE Commun. Surv. Tut., vol. 17, no. 2, pp. 757-789, Secondquarter 2015.

[5] L. R. Varshney, "Transporting information and energy simultaneously," in Proc. IEEE Int. Symp. Info. Theory, July 2008, pp. 1612-1616.

[6] P. D. Diamantoulakis and G. K. Karagiannidis, "Maximizing proportional fairness in wireless powered communications," IEEE Wireless Commun. Lett., vol. 6, no. 2, pp. 202-205, April 2017.

[7] P. D. Diamantoulakis, K. N. Pappi, Z. Ding, and G. K. Karagiannidis, "Wireless-powered communications with non-orthogonal multiple access," IEEE Trans. Wireless Commun., vol. 15, no. 12, pp. 8422-8436, Dec. 2016.

[8] G. He, L. Li, X. Li, W. Chen, L. L. Yang, and Z. Han, "Secrecy sum rate maximization in NOMA systems with wireless information and power transfer," in Proc. 9th Int. Conf. Wireless Commun. and Sig. Process. (WCSP), Oct 2017, pp. 1-6.

[9] N. Jindal, S. Vishwanath, and A. Goldsmith, "On the duality of gaussian multiple-access and broadcast channels," IEEE Trans. Inf. Theory, vol. 50, no. 5, pp. 768-783, May 2004.

[10] D. W. K. Ng, E. S. Lo, and R. Schober, "Robust beamforming for secure communication in systems with wireless information and power transfer," IEEE Trans. Wireless Commun., vol. 13, no. 8, pp. 4599-4615, Aug. 2014.

[11] J. Tang, D. K. C. So, A. Shojaeifard, K. K. Wong, and J. Wen, "Joint antenna selection and spatial switching for energy efficient MIMO SWIPT system," IEEE Trans. Wireless Commun., vol. 16, no. 7, pp. $4754-4769$, July 2017.

[12] S. Boyd and L. Vandenberghe, Convex Optimization. Cambridge University Press, Cambridge, UK, 2004.

[13] W. Dinkelbach, "On nonlinear fractional programming," Management Science, vol. 13, pp. 492-498, Mar. 1967.

[14] S. Boyd, Branch and Bound Methods. Stanford University.

[15] J. Tang, D. K. C. So, E. Alsusa, and K. A. Hamdi, "Resource efficiency: A new paradigm on energy efficiency and spectral efficiency tradeoff," IEEE Trans. Wireless Commun., vol. 13, no. 8, pp. 46564669, Aug. 2014.

[16] X. Zhou, R. Zhang, and C. K. Ho, "Wireless information and power transfer in multiuser OFDM systems," IEEE Trans. Wireless Commun., vol. 13, no. 4, pp. 2282-2294, April 2014. 